\title{
Le multilinguisme en Europe
}

Multilingualism in Europe

El multilingüismo en Europa

\section{Leonard Orban}

\section{OpenEdition}

\section{Journals}

Édition électronique

URL : http://journals.openedition.org/ries/358

DOI : $10.4000 /$ ries.358

ISSN : 2261-4265

Éditeur

Centre international d'études pédagogiques

Édition imprimée

Date de publication : 1 avril 2008

Pagination : $37-45$

ISBN : 978-2-85420-572-5

ISSN : 1254-4590

Référence électronique

Leonard Orban, "Le multilinguisme en Europe », Revue internationale d'éducation de Sèvres [En ligne]

47 | avril 2008, mis en ligne le 24 juin 2011, consulté le 30 avril 2019. URL : http://

journals.openedition.org/ries/358; DOI : 10.4000/ries.358

(c) Tous droits réservés 


\section{Le multilinguisme en Europe}

\section{Leonard Orban}

\section{LES ENJEUX}

Le paysage linguistique de l'Union européenne est très varié. Outre les vingt-trois langues officielles, sur le territoire des vingt-sept États membres, on recense environ soixante langues locales et un grand nombre de langues extérieures parlées par les communautés de migrants. Pour de nombreux habitants de notre continent, le multilinguisme fait partie de la réalité quotidienne.

Le terme de multilinguisme désigne à la fois la capacité d'une personne à utiliser plusieurs langues et la coexistence de plusieurs communautés linguistiques dans une zone géographique donnée. Ainsi défini, il est aisé d'adhérer à l'idée que la capacité de comprendre et d'utiliser plusieurs langues est une aptitude souhaitable pour tous les citoyens européens.

Nos différentes langues n'ont pas toutes le même parcours historique, ni le même rayonnement, mais elles ont toutes une légitimité et une égale dignité. Elles reflètent nos cultures et nos identités. Mais les langues sont également le premier outil de communication pour interagir avec les autres et c'est à travers elles que nous sommes à même d'échanger, de nous comprendre et d'accéder aux autres cultures.

C'est dans cet esprit que, dès l'origine des Communautés européennes, la politique linguistique a été pensée. Aujourd'hui face à la mobilité accrue des citoyens européens, aux flux migratoires et à la mondialisation, cette diversité linguistique prend une dimension nouvelle au sein de l'Union européenne et nous sommes poussés à envisager une politique plus ambitieuse pour le multilinguisme.

Dans cette perspective, le multilinguisme contribue au double objectif d'ouvrir l'Europe vers le monde et les Européens vers eux-mêmes. De même, le multilinguisme joue un rôle central dans l'intégration des communautés migrantes qui sont présentes aujourd'hui sur le territoire européen. L'enseignement de la langue d'origine aux nouvelles générations les aide à conserver leurs racines et à maintenir un contact avec les générations plus âgées. Conscients de la valeur de leur culture, ils seront davantage capables de s'intégrer dans notre société et de contribuer au développement d'une citoyenneté européenne responsable. 


\section{DES ENSEIGNANTS COMPÉTENTS ET DES PROGRAMMES APPROPRIÉS}

La diffusion du multilinguisme exige un effort pédagogique important. Il est donc essentiel que les écoles et les établissements de formation adoptent une approche holistique de l'enseignement des langues établissant des liens appropriés entre l'enseignement de la langue maternelle et des autres langues. Les professeurs de langues ont un rôle crucial à jouer dans la création d'une Europe multilingue. Il est essentiel qu'ils disposent de l'expérience adéquate dans l'utilisation de la langue cible et dans la compréhension de la culture qui y est associée.

En ce qui concerne l'enseignement scolaire, le modèle que la Commission recommande est celui de l'apprentissage de la langue maternelle plus deux autres langues, et ce, dès le plus jeune âge. Or, l'apprentissage précoce des langues nécessite des moyens spécifiques : les enseignants doivent être spécialement formés à enseigner les langues à de très jeunes enfants, les classes doivent comprendre un effectif limité pour permettre un apprentissage efficace, le matériel pédagogique doit être adapté à cette catégorie d'apprenants et une part suffisante du programme d'études doit être consacrée aux langues. Qu'elles soient prises au niveau national ou local, les initiatives visant à rendre l'apprentissage des langues accessible à un groupe d'élèves de plus en plus jeunes doivent aller de pair avec l'affectation de moyens appropriés, y compris des moyens pour la formation des enseignants.

Les compétences acquises par le biais de l'enseignement initial doivent être renforcées par d'autres méthodes. L'accent pourrait aujourd'hui être mis davantage sur l'apprentissage des langues chez l'adulte, sur l'ouverture du cercle des parties prenantes au secteur des entreprises, sur la formation professionnelle continue et enfin sur l'apprentissage informel des langues au travers des médias et des activités culturelles.

L'apprentissage des langues doit être «vivant» et, si les connaissances académiques sont essentielles, les apprenants doivent également acquérir de bonnes capacités de communication, c'est-à-dire des compétences actives plutôt que des connaissances passives. L'objectif n'est pas forcément d'atteindre la même fluidité qu'un locuteur natif, mais de parvenir à un niveau adéquat de compréhension à la lecture et à l'audition et de pouvoir s'exprimer à l'écrit et à l'oral dans deux langues étrangères.

Ainsi, en dehors du cadre scolaire, de multiples options sont possibles, selon l'objectif que l'on veut atteindre. S'approprier une langue peut se faire d'une manière variée et à des niveaux différents de compétences.

L'apprentissage doit aussi se diversifier et ne pas se limiter à l'anglais. Une plus grande variété linguistique est nécessaire pour promouvoir l'intégration européenne et le dialogue avec les groupes de migrants. 
Des activités culturelles centrées sur la musique, la littérature, le théâtre ou les films étrangers, des vacances à l'étranger, des activités de jumelage ou un service volontaire à l'étranger peuvent se révéler des occasions idéales de se familiariser avec d'autres langues et d'autres cultures.

\section{LES PROGRAMMES COMMUNAUTAIRES POUR L'APPRENTISSAGE DES LANGUES}

L'engagement de la Commission européenne en faveur de l'apprentissage des langues et de la diversité linguistique est déjà ancien. Le premier programme complet en faveur de l'enseignement et de l'apprentissage des langues, "Lingua», est entré en vigueur en 1989 et depuis, les langues ont toujours été au cœur des programmes européens dans le domaine de l'éducation et de la formation.

Dans la perspective de contribuer davantage au développement de la formation et de l'éducation, la Commission européenne a renouvelé son programme d'éducation et lancé en novembre 2006 le «Programme pour l'éducation et la formation tout au long de la vie». Ce programme va bien audelà des bourses Erasmus, largement médiatisées, et compte de nombreuses composantes qui touchent directement les langues. L'objectif du «Programme d'action dans le domaine de l'éducation et de la formation tout au long de la vie 2007-2013» est de développer et de renforcer les échanges, la coopération et la mobilité afin que les systèmes d'éducation et de formation deviennent une référence de qualité mondiale conformément à la stratégie de Lisbonne. Le programme vise ainsi à contribuer au développement de l'Union européenne comme société de la connaissance avancée, caractérisée par un développement économique durable accompagné d'une amélioration quantitative et qualitative de l'emploi et d'une plus grande cohésion sociale.

Au-delà de cet objectif général, le programme poursuit des objectifs spécifiques concernant l'éducation et la formation tout au long de la vie, en contribuant en particulier au développement d'un enseignement et d'une formation de qualité ainsi qu'à la promotion d'un niveau de performance élevé, de l'innovation et de la dimension européenne des systèmes et pratiques en vigueur.

Le programme a pour but d'encourager la réalisation d'un espace européen de l'éducation et de la formation tout au long de la vie, d'améliorer la qualité, l'attrait et l'accessibilité des possibilités d'éducation et de formation, de renforcer la cohésion sociale, la citoyenneté active, le dialogue interculturel, l'égalité hommes-femmes et l'épanouissement personnel.

L'éducation tout au long de la vie offre aussi des nouvelles perspectives en matière de créativité, de compétitivité et de capacité d'insertion professionnelle. Elle contribue à l'accroissement de l'implication active des personnes de 
tous âges, y compris les personnes ayant des besoins particuliers et les groupes défavorisés. Le programme cherche à permettre une utilisation optimale des résultats, des produits et processus innovants ainsi que l'échange de bonnes pratiques.

Le Programme pour l'éducation et la formation tout au long de la vie prévoit le financement de projets linguistiques au titre des actions Comenius et Grundtvig, pour offrir aux jeunes élèves de véritables possibilités d'utiliser leurs compétences linguistiques en établissant des contacts avec des élèves de leur âge et aux adultes la possibilité d'une formation permanente.

De l'enseignement préscolaire aux établissements du secondaire, le programme «Comenius» a pour objectif de promouvoir la compréhension des différentes cultures européennes grâce aux échanges et à la coopération entre les écoles de différents pays. Le programme s'adresse à la communauté éducative au sens large, notamment aux autorités locales, aux associations de parents d'élèves ou aux instituts de formation des enseignants. Comenius vise à accroître la qualité de l'enseignement scolaire, à renforcer sa dimension européenne et à promouvoir la mobilité, l'apprentissage des langues et l'intégration. Ce programme soutient les partenariats entre écoles dans des domaines thématiques présentant un intérêt commun, ainsi que des projets multilatéraux novateurs dans le domaine des méthodes pédagogiques ou des programmes d'études. Plus particulièrement, Comenius a pour objectif de promouvoir la participation d'au moins trois millions d'élèves à des activités éducatives communes d'ici à 2013.

Destiné à répondre aux défis posés par la nécessité de la mise à jour des connaissances, le programme "Grundtvig» a pour objectif de fournir aux adultes les moyens d'améliorer leur savoir-faire et leurs compétences, et de leur permettre ainsi de s'adapter aux mutations du marché du travail et de la société. Grundtvig est axé sur toutes les formes d'éducation permanente non professionnelle pour adultes. Il s'adresse à tous ceux et celles qui veulent apprendre, aux enseignants, aux formateurs et autres intervenants du secteur de la formation des adultes, ainsi qu'aux institutions. Les associations de formation des adultes, les services d'orientation et d'information, les ONG, les entreprises, les centres de recherche et les établissements d'enseignement supérieur ont la possibilité de collaborer par le biais de partenariats transnationaux, de projets et de réseaux européens.

Le programme Erasmus apporte bien sûr une contribution majeure à l'apprentissage des langues. Il s'agit d'un effet collatéral non négligeable de ce programme bien connu du grand public.

L'apprentissage intégré d'un contenu et d'une langue («EMILE») apporte lui aussi une contribution majeure à la réalisation des objectifs de l'Union européenne en termes d'apprentissage des langues. Cette méthode permet aux élèves d'utiliser directement leurs nouvelles compétences linguis- 
tiques, plutôt que de se concentrer d'abord sur l'apprentissage avant de passer à la pratique. Elle ouvre les portes de l'apprentissage des langues à un plus large éventail d'élèves. Elle offre une exposition aux langues sans requérir des heures de cours supplémentaires, ce qui peut s'avérer particulièrement intéressant dans un environnement professionnel.

\section{LES POLITIQUeS Éducatives}

$\mathrm{Au}$ niveau européen, il faut noter une accélération notable de la politique du multilinguisme au cours de ces dernières années.

À l'issue de l'Année européenne des langues, en 2001, le Parlement européen et le Conseil ont adopté des résolutions invitant la Commission européenne à prendre d'autres mesures pour promouvoir les langues. En 2002, les chefs d'État et de gouvernement réunis à Barcelone ont pris l'engagement politique d'améliorer la maîtrise des compétences de base, en particulier en prévoyant l'enseignement de deux langues étrangères pour tous dès le plus jeune âge.

En mars 2002, à Barcelone, les chefs d'État et de gouvernement ont aussi relevé le manque de données concernant les compétences linguistiques réelles des citoyens et ont prôné l'élaboration d'un indicateur européen de compétence linguistique. Cet indicateur fournira de précieuses informations aux décideurs des systèmes d'éducation et de formation. La Commission travaille à l'heure actuelle à la conception et à la gestion d'un test périodique des compétences linguistiques qui rassemblera des données pour un nouvel indicateur européen de compétence linguistique.

En 2003, le Parlement européen a adopté un rapport d'initiative invitant la Commission à mieux tenir compte des langues régionales et moins répandues dans le contexte de l'élargissement et de la diversité culturelle.

Pour répondre aux demandes du Conseil et au Parlement européen, la Commission a adopté pour 2004-2006 le plan d'action Promouvoir l'apprentissage des langues et la diversité linguistique. Les conclusions du rapport de mise en œuvre de ce plan d'action ont établi qu'au cours des cinq dernières années, les Etats avaient progressivement réformé leur enseignement primaire, et dans certains cas préscolaire, pour pouvoir enseigner plus tôt une langue étrangère.

\section{Le programme de la Commission européenne en matière de promotion du multilinguisme}

Sous l'impulsion du Président Barroso, le développement de la politique du multilinguisme a encore été renforcé avec la création en janvier 2007 d'un portefeuille spécifique, dont j'ai la charge. L'idée est d'élargir l'action 
de la Commission à d'autres domaines qui ne sont pas forcément rattachés à la question de la formation et de lier de façon transversale cette problématique avec toutes celles qui sont gérées au niveau communautaire.

Au cours de cette année, nous nous sommes efforcés de mettre en avant les implications économiques du multilinguisme. La Commission a souhaité sensibiliser le monde des affaires sur l'impact des compétences linguistiques sur les performances des entreprises et sur le potentiel de croissance qu'une meilleure maîtrise des langues étrangères induit.

Avec l'étude «Multilinguisme et compétitivité des entreprises» réalisée pour la Commission en 2006 par le CILT, le Centre national britannique des langues, il a été démontré que chaque année, des milliers d'entreprises européennes passent à côté d'opportunités commerciales et de contrats du fait de leur manque de compétences linguistiques.

Selon la même étude, l'anglais demeure assurément la première lingua franca du commerce international, mais la demande est en augmentation pour d'autres langues. Si environ un quart des entreprises sur lesquelles a porté l'étude estiment qu'elles doivent encore «améliorer leur anglais», elles sont tout aussi nombreuses à penser que l'anglais ne suffit pas au commerce mondial et qu'elles doivent renforcer leurs capacités linguistiques en allemand et en français, voire en espagnol et en russe, pour ne mentionner que quatre des langues les plus souvent citées. Beaucoup d'entreprises - les grandes, en particulier signalent en outre qu'elles ont besoin de langues non européennes, telles que le chinois, l'arabe ou l'ourdou, dès lors qu'elles cherchent à se développer hors d'Europe.

L'étude conclut qu'il existe, pour les petites entreprises en Europe, un potentiel considérable d'accroissement de leurs exportations totales si elles consentent à investir davantage dans les langues et à élaborer des stratégies linguistiques cohérentes. Les entreprises qui renforcent leurs compétences linguistiques sont mieux en mesure d'exploiter les possibilités qu'offre le marché intérieur de l'Union qui, avec près d'un demi-milliard de personnes, est le plus important du monde.

Une autre étude, «ELAN : Incidences du manque de compétences linguistiques des entreprises sur l'économie européenne», a permis de mettre en évidence l'existence d'un rapport direct entre langues et bons résultats à l'exportation. Plus précisément, elle distingue à cet égard quatre mesures de «gestion linguistique»: adoption d'une stratégie de communication multilingue, recrutement de locuteurs natifs, recrutement de personnel possédant des compétences linguistiques et recours à des traducteurs et à des interprètes. Chaque PME du secteur de l'exportation pourrait engranger des profits plus que substantiels si elle appliquait une ou plusieurs de ces mesures.

C'est dans cette optique que la Commission a organisé, le 21 septembre 2007, la conférence «Les langues font nos affaires», qui a réuni plus de 
250 représentants d'entreprises, de chambres de commerce, d'instituts culturels, d'écoles de langues et d'associations commerciales, ainsi que des responsables politiques et d'autres parties prenantes.

Cet événement a été suivi par le lancement d'un Forum des entreprises sur le multilinguisme, présidé par Etienne Davignon, président de CSR Europe, qui déterminera de quelle façon les entreprises peuvent utiliser le multilinguisme pour optimiser leurs résultats.

La Commission explore les possibilités de développement du multilinguisme aussi dans le cadre de la traduction et de l'interprétation.

Une étude sur les nouvelles technologies et la diversité linguistique est envisagée en 2008 dans le but d'évaluer le potentiel d'intérêt des nouvelles technologies pour attirer et former les étudiants.

La promotion de nouveaux instruments de communication multilingue est aussi prise en compte, dans la perspective de développer des activités de recherche dans le domaine de l'intelligence artificielle pour des utilisations dans la traduction et l'interprétation.

L'ouverture au public de la base de données terminologique IATE (Inter-Active Terminology for Europe) fournit un soutien aux entreprises en leur donnant accès à une terminologie précise et cohérente dans les domaines techniques et juridiques.

Il faut aussi mentionner les efforts que la Commission met en ouvre pour atteindre elle-même son objectif de multilinguisme et d'accessibilité aux citoyens de l'Union, par l'amélioration des versions multilingues sur ses sites web.

Toujours dans l'idée d'associer la politique du multilinguisme à d'autres politiques de l'Union, le programme «Culture»(2007-2013) prévoit une aide à la traduction littéraire dans les langues de l'Union. Grâce à cette subvention, plusieurs milliers de livres ont été traduits, rendant accessibles des œuvres littéraires à un plus vaste public européen et favorisant de la sorte la diversité culturelle et la diffusion des langues moins connues.

Dans le domaine culturel, l'année 2008 a été consacrée comme année européenne du dialogue interculturel. Bien entendu, dans ce cadre aussi, les langues ont un rôle clé à jouer. La diffusion des langues contribue à la percée des idées et de l'innovation. La connaissance réciproque indispensable pour développer un sentiment d'appartenance commune passe par le partage de la langue. Les langues des communautés de migrants font aussi aujourd'hui partie du paysage linguistique européen. Pour les migrants, elles sont un instrument de prise de conscience de leurs propres racines, de valorisation de leur culture d'origine et de communication entre générations. Pour les citoyens européens, elles sont une porte d'accès au monde. Ainsi, un groupe d'intellectuels présidé par l'écrivain Amin Maalouf a été mis en place dans cet esprit. Ce groupe a pour mandat de définir la contribution du multilinguisme à l'Année européenne du dialogue interculturel en 2008. Son rapport a été rendu public au début de l'année 2008. 
Enfin, je souhaite mentionner qu'une communication spécifique sur le multilinguisme est en cours de préparation et devrait être adoptée par la Commission en septembre 2008. Avec ce document d'une grande portée politique et symbolique, il s'agira de donner un nouvel élan et une nouvelle direction à notre action en faveur des langues.

Pour nourrir notre réflexion, nous avons, dans un premier temps, consulté des groupes sectoriels, les administrations des États membres et les entreprises et dans un deuxième temps, lancé une consultation en ligne pour laquelle 2500 réponses ont été reçues. Le rapport rendu par un groupe de haut niveau pour le multilinguisme, constitué spécialement à cet effet, constitue également un apport fondamental aux réflexions qui vont mener à cette communication.

\section{Effets attendus}

Le développement de la politique linguistique est essentiel au bon fonctionnement de l'Union européenne. Renforcer les compétences linguistiques des citoyens permet de contribuer à la fois au développement économique, social et culturel de l'Union européenne. Le multilinguisme peut contribuer à une meilleure exploitation du potentiel de l'Europe en vue d'une croissance soutenue et de la création d'emplois plus nombreux et de meilleure qualité. Le multilinguisme peut également favoriser les contacts entre Européens et encourager le développement d'une citoyenneté européenne.

Plus les Européens seront en mesure de parler d'autres langues, outre leur langue maternelle, plus ils pourront échanger dans les débats sur leur avenir commun, aborder leurs problèmes d'une manière cohérente, saisir de nouvelles opportunités, participer activement à la vie politique et sociale, et donc, plus efficacement au bien-être et au progrès de la société européenne. 